\title{
EVALUACION DE RIBERA Y HABITAT FLUVIAL A TRAVES DE LOS INDICES QBR E IHF
}

\section{ASSESSMENT OF A RIPARIAN AND FLUVIAL HABIT THROUGH QBR AND IHF INDEX}

\author{
Alejandro Palma ${ }^{1}$, Ricardo Figueroa ${ }^{2} \&$ Víctor H. Ruiz ${ }^{3}$ \\ ${ }^{1}$ Departamento de Ecología, Facultad de Biología, Pontificia Universidad Católica de Chile, Casilla 113-D, Santiago, \\ Chile.lapalma@bio.puc.cl \\ ${ }^{2}$ Unidad de Sistemas Acuáticos, Centro de Ciencias Ambientales EULA-Chile, Universidad de Concepción, Chile. \\ Casilla 160-C, Concepción, Chile.rfiguero@udec.cl \\ ${ }^{3}$ Departamento de Zoología, Facultad de Ciencias Naturales y Oceanográficas. Universidad de Concepción, Chile. \\ Casilla 160-C, Concepción, Chile. vruiz@udec.cl
}

\begin{abstract}
RESUMEN
El desarrollo de métodos para evaluar de manera rápida y efectiva un sistema hidrográfico, pero sin perder la capacidad de integrar información de la salud del sistema, ha despertado un alto interés en el último tiempo. El presente estudio evaluó la condición ecológica de la parte alta del estero Nonguén (primero cinco kilómetros) durante dos periodos estacionales, evaluando 5 transectos de 100 metros, separadas aproximadamente por un kilómetro. Para ello se aplicaron dos índices desarrollados para climas mediterráneos en la península ibérica: el índice de calidad de ribera (QBR) y el índice de hábitat fluvial (IHF). Los resultados indican que estos índices entregan una aproximación confiable de la calidad de la ribera del río y del cauce mismo. Se discute sobre su adaptabilidad para sistemas fluviales en general.
\end{abstract}

Palabras ClaVes: QBR, IHF, mediterráneo, Chile.

\section{ABSTRACT}

The development of methods that allow us to evaluate quickly and efficiently a freshwater system has been of high interest in the last time. This work evaluated the ecological condition of the headwater of the Nonguén stream (first five kilometers) during two seasonal periods, evaluating 5 transect of $100 \mathrm{~m}$. separate by one kilometers each other. Two indexes development to mediterranean climate in the Iberian Peninsula was appliqued: the QBR (to evaluate riparian quality) and IHF (to evaluate physical habitat) index. The results shows the efficiency of these indexes like rapid assessments of environmental quality to shows a first trustworthy approximation of the hydrological system. The application of these indexes to freshwater system in general are discuss.

KeYwords: QBR, IHF, mediterranean, Chile.

\section{INTRODUCCION}

El desarrollo de métodos que nos permitan evaluar de manera rápida y efectiva el estado ecológico de los sistemas fluviales ha despertado un alto interés en el último tiempo, siendo inclusive uno de los objetivos de la Directiva Marco del Agua de la Comunidad Europea (D.O.C.E., 2000). Esta situación es compleja cuando debemos definir los parámetros a considerar, considerando que a medida que avanzamos en la comprensión del comportamiento de ríos y arroyos, lo estamos haciendo sobre sistemas sujetos a una degradación y/o modificación de su estado natural (Allan 1997; 2004). Por esta razón resulta indudable que se requiere de métodos que orienten a la gestión para mantener y restaurar estos complejos sistemas.

Bajo esta premisa se han desarrollado técnicas 
y métodos de muestreo en ríos para evaluar la calidad biológica en función de los macroinvertebrados (e.g. IBF, BMWP) (Barbour et al. 1999; Bonada et al. 2002) y de su entorno (aspectos físicos del cauce y de la ribera) mediante el uso de índices especialmente diseñados para ello: el índice de Hábitat Fluvial (IHF) y el índice de Bosque de Rivera (QBR) (Pardo et al. 2002; Suarez et al. 2002) desarrollados para ambientes mediterráneos. La información obtenida a través de estos últimos son de gran relevancia al considerar que un buen estado del entorno natural incide de manera decisiva en la calidad ecológica del cauce en términos de calidad de agua y biota asociada (Bonada et al. 2002).

Las zonas de ribera con abundante vegetación nativa, han sido reconocidas como áreas de importancia ecológica, no sólo por el aporte de material orgánico al sistema (Cummins 1974; Lemly \& Hilderbrand 2000; Jonsson et al. 2001), sino también por una serie de otras funciones entre las cuales destacan: crear un ambiente propicio para especies que se alimentan de hojas, como para la flora bacteriana y los hongos que facilitan la degradación de material orgánico (Cummins 2002); son corredores de interacción entre animales terrestres y acuáticos (Faush et al. 2002; Ward et al. 2002); forman un ecotono de interacción de los ecosistemas terrestre/acuático, donde los flujos de energía y nutrientes permiten una fuerte interacción entre estos ecosistemas (Wallace et al. 1997; Ríos \& Bailey, 2006); es una importante zona de refugio, reproducción y cría de aves (Gergel et al. 2002); permite zonas de mayor sombras en las cabeceras de los ríos, las que ayudan a mantener la temperatura en verano dentro de rangos tolerables para especies adaptadas a aguas frías (Bunn et al. 1999; Abel \& Allan 2002; Cummins 2002). Pero sin duda, la mayor relevancia es la protección que brinda al sistema acuático, al actuar como trampas naturales (zona buffer) para retener sedimentos, nutrientes y otros contaminantes desde los suelos adyacentes a los cursos de agua (Gergel et al. 2002; Baudry \& Thenail, 2003). Compuestos utilizados en la agricultura (especialmente organoclorados, PCBs y sustancias asociadas a nutrientes que contienen nitrógeno, fósforo y sulfatos) pueden ser degradados en condiciones anaeróbicas a elementos menos tóxicos (La Greda 1996; Mariuzzo \& Scamparini, 1999; Kao \& Wang 2000) disminuyendo el riesgo para el componente biológico del sistema acuático.

Entonces, la evaluación del entorno en términos de su naturalidad es de gran relevancia ecosistémica y puede ser fácilmente determinada a través de estos índices (QBR e IHF) que no solo son integrativos, sino que permiten establecer protocolos de trabajos que siendo replicables, facilitan las comparaciones de distintos grupos de trabajo de manera de ajustar y estandarizar las metodologías propuestas, siendo por tanto una interesante herramienta de gestión de los recursos hídricos. El objetivo de este trabajo es aportar evidencia de la posible aplicabilidad de estos índices en otras partes del mundo que son comparables (i.e. en clima mediterráneo), siendo este el primer estudio en un sistema fluvial mediterráneo sudamericano.

\section{METODOLOGIA}

AREA DE ESTUDIO:

El área de estudio, corresponde al Estero Nonguén, una subcuenca costera tributaria del curso inferior del río Andalién, ubicada entre los $36^{\circ} 49^{\prime}$ y los $36^{\circ}$ $54^{\prime} \mathrm{S}$ y $\operatorname{los} 72^{\circ} 57^{\prime}$ y $\operatorname{los} 73^{\circ} 01^{\prime} \mathrm{W}$, con una orientación general de sur a norte, recorriendo una distancia de más de $15 \mathrm{~km}$ dando forma a una cuenca de $44 \mathrm{~km}$ de superficie y con un caudal medio de $1 \mathrm{~m}^{3} \mathrm{~s}^{-1}$. Ha sido utilizado como fuente de aprovisionamiento de agua potable en las últimas décadas (Jaque, 1994), por lo que su acceso restringido ha manteniendo un estado relativamente natural, ofreciendo un excelente lugar para la realización de estos estudios. El presente estudio fue realizado durante los períodos primavera-verano y otoño-invierno entre los años 2003 - 2004, en donde se consideraron 5 estaciones de muestreo en los primeros $5 \mathrm{~km}$ del sistema: dos en un área de rápidos (E1 y E2) aguas arriba, una en un área intermedia (E3) y dos en un área de llanura (E4 y E5), las cuales están separadas a una distancia aproximada de $1 \mathrm{~km}$ entre ellas (Figura 1).

\section{INDICES DE LA CALIDAD DEL ENTORNO}

QBR: Siguiendo a Suarez et al. (2002) se utiliza el QBR (Índice de Calidad del Bosque de Ribera) propuesto por Munné et al. (1998; 2003), que en cuatro bloques recoge distintos componentes y atributos de las riberas: cubierta vegetal, estructura de la vegetación, naturalidad y complejidad del bosque ribereño y grado de alteración del canal fluvial. Los valores del índice se distribuyen en cinco rangos de calidad: >95: estado natural, bosque de ribera sin alteraciones; 90-75: calidad buena, bosque ligeramente perturbado; 70-55: 
calidad aceptable, inicio de alteración importante; 3050: calidad mala, alteración fuerte; $<25$ : calidad pésima. Degradación extrema.

$I H F$ : De igual manera se utiliza el IHF (Índice de Hábitat Fluvial) (Pardo et al. 2002) que consta de siete bloques o apartados en los que se valora de manera independiente la presencia de distintos componentes en el cauce fluvial, entre ellos aspectos físicos del cauce relacionados con la heterogeneidad de hábitats y que dependen en gran medida de la hidrología y del sustrato existente. Entre ellos, la frecuencia de rápidos, la existencia de distintos regímenes de velocidad y profundidad, el grado de inclusión y sedimentación en pozas, y la diversidad y representación de sustratos. También se evalúa la presencia y dominancia de distintos elementos de heterogeneidad, que contribuyen a incrementar la diversidad de hábitat físico y de las fuentes alimenticias, entre ellos materiales de origen alóctono (hojas, madera) y de origen autóctono, como la presencia de diversos grupos morfológicos de productores primarios. La puntuación final del índice es el resultado de la suma de la puntuación obtenida en cada uno de los bloques y nunca puede ser mayor que 100 .

Los detalles metodológicos y las hojas de campo para el uso del QBR e IHF se encuentran en Jáimez-Cuéllar et al. (2002) y pueden ser obtenidos en el protocolo ECOSTRIMED (http://www.diba.es/mediambient/ es/protocol.asp), el cual ha sido desarrollado por un grupo multidisciplinario coordinado por la Universidad de Barcelona para evaluar la calidad ecológica de los ríos mediterráneos y que se ajuste a las prioridades ambientales de la Directiva marco del agua de la Comunidad Europea.

Los resultados obtenidos por estos índices fueron comparados por los obtenidos con las comunidades biológicas durante el mismo periodo, según Figueroa et al. (2005) figura 2.

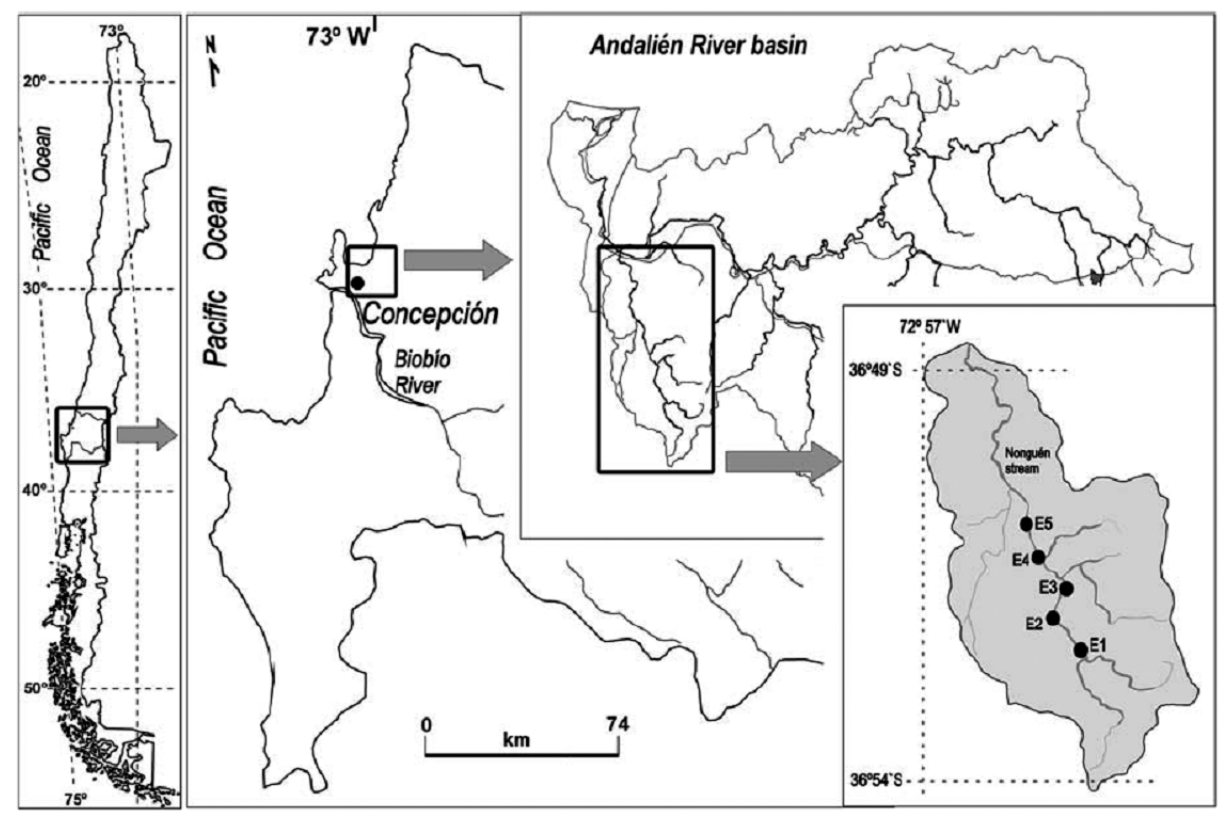

Figura 1. Area de estudio y localización de las estaciones de muestreo (E1-E5).

Figure 1. Study area and location of sampling stations (E1-E5). 


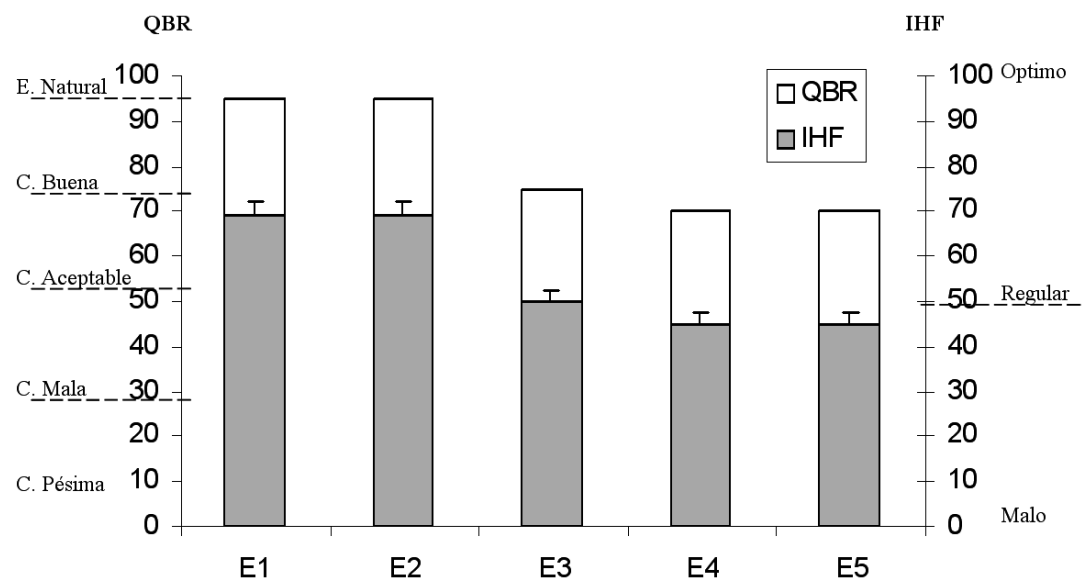

FigURA 2. QBR e IHF obtenidos en las estaciones de muestreos indicando la desviación.

FIGURE 2. QBR and IHF obtained in all sampling stations showing the desviation.

\section{RESULTADOS}

QBR: Los valores del índice se distribuyeron de la siguiente forma: E1 y E2 "estado natural" (QBR>95), E3 “calidad buena" (QBR>75), E4 y E5 "calidad aceptable" $(\mathrm{QBR}>50)$ estableciendo una tendencia a la degradación de la ribera aguas abajo del Estero (Figura 2). El área de estudio también presenta una disminución en la zona de inundación y en el grado de cubierta vegetal de la ribera a medida que nos alejamos de la parte alta del cauce. IHF: Los valores de este índice fueron 70 para E1 y E2, 50 para E3 y finalmente 45 para E4 y E5 (Figura 2). Si bien los componentes del IHF (regímenes de velocidad/ profundidad, frecuencia de rápidos, diversidad de sustrato) puntúan más en invierno y otoño la cobertura de vegetación acuática y porcentaje de sombra en el cauce son similares a lo largo del año. Las estaciones E4 y E5 en general puntuaron mas bajo debido al porcentaje de sedimentación observado y la baja frecuencia de rápidos que entregaron una diferencia notoria con las demás estaciones. Con todo, los valores de IHF en ambos períodos muestreados resultaron ser similares en su puntuación general (con valores 5) para cada estación (ver Figura 2).
Las comunidades biológicas entregan el mismo patrón al clasificar las estaciones de muestreo para el estero Nonguén, agrupa a las estaciones E1 y E2, separa a E3, y agrupa por otro lado a E4 y E5 (ver Figueroa et al. 2005, Figura 2).

\section{DISCUSION}

El uso de los índices de calidad del entorno (QBR e IHF) mostraron una clara idea de cómo se encontraban comparativamente las distintas estaciones estudiadas, puesto que ambos fueron mostrando una menor puntuación aguas abajo pero siempre manteniendo una "buena calidad" en las cinco estaciones. Ejemplo de lo anterior es la cabecera del sistema, la que mantiene un relicto del bosque caducifolio de Concepción caracterizado por especies de Nothofagus, particularmente $N$. dombeyi y $N$. oblicua, y de elementos representativos de la flora regional como Criptocarya alba, Aetoxicon punctatum, Peumus boldus, Persea lingue, Podocarpus saligna y Laurelia sempervirens (Eula 2002), sector que mostró las más altas puntuaciones, calificando como "estado natural" dentro de las categoría de estos índices.

Aguas abajo el entorno comienza a variar, y con 
ellos las puntuaciones muestran un leve descenso tanto en el hábitat como en la ribera, lo que podría ser esperable al pasar de una zona de rápidos a una de llanura, y en donde comienza a vislumbrarse las condiciones existentes aguas abajo del sitio de este estudio, (parte media y baja del estero), donde se aprecian primeramente áreas de cultivos con plantaciones y posteriormente un ambiente netamente urbano en su desembocadura (Habit et al. 2003). Así, el uso de los índices IHF y QBR mostraron una gran utilidad y efectividad a la hora de evaluar de manera rápida y objetiva la calidad del entorno como primera medida de salud. Lo anterior adquiere mayor relevancia si comparamos estos resultados con los entregados por las comunidades biológicas presentes en las mismas estaciones en el área de estudio, observándose el mismo patrón mediante los tres métodos al clasificar las estaciones de muestreo para el estero Nonguén: se agrupan las estaciones E1 y E2, se separa E3, y se agrupan por otro lado E4 y E5. Esto sugiere una alta correlación entre los resultados entregados por los índices de calidad de entorno aquí evaluados (QBR e IHF) y los que entregan las comunidades de macroinvertebrados presentes en el lugar, evidenciando además la estrecha relación existente entre los factores ambientales y los biológicos, situación ampliamente demostrada por varios autores (Cummins 1974; Rabeni \& Minshall, 1977; Voelz \& McArthur 2000; Baer et al. 2001; Figueroa et al. 2006).

La utilización de índices de calidad, ya sea del entorno o de tipo biológicos usando macroinvertebrados bentónicos (Figueroa et al. 2007), resultan de gran utilidad debido a su bajo costo, su fácil estandarización y sobre todo por la facilidad que ofrecen a grupos de trabajos no especializados en la obtención de resultados eficaces y reales de lo que sucede en los sistemas de aguas corrientes, lo cual es uno de los objetivos de los Protocolos Rápidos de Evaluación de la Calidad Ecológica.

Identificar la calidad del entorno físico y poder valorarlo cuantitativamente es uno de los objetivos de la comunidad Europea dentro de la Directiva Marco del Agua (D.O.C.E. 2000), así como determinar el estado ecológico de sus aguas continentales basándose en criterios biológicos (Jaímez-Cuéllar et al. 2002). Diversas actividades humanas como asentamientos humanos, actividades industriales, deforestación, desarrollo de agricultura y pastoreo, han provocado una fuerte degradación de estos ambientes. Los resultados son incrementos en la sedimentación, formación de bancos de arenas con la consecuente alteración de la geomorfología y hábitats ribereños, pérdida de diversidad de especies, estructura y composición de macroinvertebrados, alteración de régimen hidrológico (Naiman et al. 1993; Ríos \& Bailey, 2006; Innis et al. 2000). El reconocer y clasificar el alto grado de modificación de estos sistemas dentro de un marco biogeográfico es el objetivo final de este tipo de herramientas (Barbour et al. 1999; Bonada et al. 2002). No obstante, pequeñas modificaciones y adaptaciones locales de estos siguen siendo una alternativa eficaz para aplicar en otras regiones.

En este sentido, el área de estudio del presente trabajo se encuentra inmersa dentro de un hábitat igualmente mediterráneo. Durante el verano el nivel de los caudales baja, dejando un pequeño canal de agua que se mantiene gracias al agua de escasos deshielos, pero principalmente al agua retenida en el bosque nativo de la parte alta de la cuenca. Durante el invierno en cambio el río eleva sus caudales, con eventos importantes que pueden modificar sustancialmente las condiciones en el hábitat. Por tanto, la adaptación de algunos índices como el QBR e IHF, pueden ser una herramienta eficaz en nuestros sistemas fluviales mediterráneos, donde los resultados de este estudio muestran la eficacia del uso de estos índices y servirían para entregar una primera aproximación confiable de lo que ocurre en el sistema hídrico.

Finalmente es importante destacar que sería interesante desarrollar índices similares para ríos de zonas lluviosas templadas (e.g. $38^{\circ} \mathrm{S}$ a $\left.56^{\mathrm{a}} \mathrm{S}\right)$, dado que estos índices no aplicarían en esos casos debidos a las diferencias en los regímenes de caudales y estructuras de las cuencas en general.

\section{AGRADECIMIENTOS}

Este trabajo ha sido parcialmente financiado por la dirección de investigación de la Universidad de Concepción, Chile (Proyecto DIUC $\mathrm{N}^{\circ}$ 205.310.048-1).

\section{BIBLIOGRAFIA}

Abell, R. \& J.D. Allan. 2002. Riparian shade and stream temperatures in an agricultural catchment, Michigan, USA. Verthandlundgen der internationale Vereinigung für theoretische und angewandte Limnologie, 28:232-237. 
Allan, J. D. 1997. Stream Ecology. Structure and function of running waters. Chapman \& Hall. 388 pp.

Allan, J.D. 2004. Landscapes and riverscapes: the infuence of land use on Stream ecosystems. Annu. Rev. Ecol. Evol. Syst. 35:257-284.

Baer, S., R. Siler, S. Eggert \& J. Wallace. 2001. Colonization and production of macroinvertebrates on artificial substrata: upstream-downstream responses to a leaf litter exclusion manipulation. Freshwater Biology 46: 347-365

Barbour, M.T., J. Gerritsen, B.D. Snyder \& J. B. STRIBLING. 1999. Rapid Bioassessment Protocols for Use in Streams and Wadeable Rivers: Periphyton, Benthic Macroinvertebrates and Fish, 2ond ed. EPA 841-B-99-002. US EPA, Office of Water, Washington D.C., USA.

Baudry J. \& C. Thenail. 2004. Interaction between farming systems, riparian zones and landscape patterns: a case study in western France. Landscape and urban planning 67: 121-129.

Bonada, N., N. Prat, A. Munné, M. Plans, C. Solà, M. Álvarez, I. Pardo, G. Moyà, G. Ramon, M. Toro, S. Robles, J. Avilés, M. L. SuÁrez, M. R. VidalAbarca, A. Mellado, J. L. Moreno, C. Guerrero, S. Vivas, M. Ortega, J. Casas, A. SánchezOrtega, P. Jáimez-Cuéllar \& J. Albatercedor. 2002. Intercalibración de la metodología GUADALMED. Selección de un protocolo de muestreo para la determinación del estado ecológico de los ríos mediterráneos. Limnetica 21: 13-33.

BunN, S.P., P.M. Davies \& T.D. Mosisch. 1999. Ecosystem measures of river health and their response to riparian and catchment degradation. Freshwater Biology 41:333-345.

Cummins, K.W. 1974. Structure and function of stream ecosystems. BioScience 24:631-641.

Cummins, K.W. 2002. Riparian-stream linkage paradigm. Verthandlundgen der internationale Vereinigung für theoretische und angewandte Limnologie, 28:49-58

D.O.C.E. 2000. Directiva 2000/60/CE del Parlamento Europeo y del Consejo de 23 de octubre de 2000 por la que se establece un marco comunitario de actuación en el ámbito de la política de aguas. D.O.C.E. L 327 de 22.12.00. 69 pp.

EULA. 2002. Diagnóstico y zonificación del Parque Metropolitano Fundo Nonguén. Informe Línea de Base, Oficina Asistencia técnica, centro de Ciencias Ambientales, EULA - Chile, Universidad de Concepción, Concepción, Chile. 258 pp.

Faush, K., M. Power \& M. Murakami. 2000. Linkages between stream and forest food webs: Shigeru Nakano's legacy for ecology in Japan. Trend Ecology and Evolution, 17(9): 429-433.

Figueroa, R., V.H. Ruiz, A. Palma \& F. Encina. 2005. Simplificación en el uso de macroinvertebrados bentónicos en la evaluación de la calidad de las aguas fluviales. Interciencia 30(12): 770-774

Figueroa, R., V.H. Ruiz, X. Neill, E. Araya \& A. Palma.
2006. Invertebrates Colonization Patterns in a Mediterranean Chilean Stream. Hydrobiologia 571: 409-417.

Figueroa, R., A. Palma \& V.H. Ruiz. 2007. Análisis comparativo de índices bióticos utilizados en la evaluación de la calidad de las aguas en un río mediterráneo de Chile: río Chillan, VIII Región. Revista Chilena de Historia Natural 80 (2):225242.

Gergel, S.E., M.G. Turner, J.R. Miller, J.M. Melack \& E.H. Stanley. 2002. Landscape indicators of human impacts to riverine systems. Aquatic Sciences 64:118-128.

Habit, E., P. Victoriano \& A. Rodriguez-Ruiz. 2003. Variaciones espacio-temporales del ensamble de peces de un sistema fluvial de bajo orden del centro-sur de Chile. Revista Chilena de Historia Natural 76:3-14.

InNIS, S.A., R.J. NAiman \& S.R. ElLIOT. 2000. Indicators and assessment methods for measuring the ecological integrity of semi-aquatic terrestrial environments. Hydrobiologia 422/423: 111-131.

Jáimez-Cuellar, P., S. Vivas, N. Bonada, S. Robles, A. Mellado, M. Álvarez, J. Avilés, J. Casas, M. Ortega, I. Pardo, N. Prat, M. Rieradevall, C. E. Sáinzcantero, A. Sánche-Ortega, M. L. SuÁrez, M. Toro, M.R. Vidal-Abarca, C. ZamoraMuñoz \& J. Alba-Tercedor. 2002. Protocolo GUADALMED (PRECE). Limnetica 21: 187-204.

JAQUE, E. 1994. Problemas ambientales en un área de expansión urbana. Cuenca del Estero Nonguén, Concepción. Revista Geográfica de Chile Terra Australis 39: 65-78.

Jonsson, M., B. Malmevist \& P. Hoffsten. 2001. Leaf litter breakdown rates in boreal streams: does shredder species richness matter? Freshwater Biology 46: 161-171.

KaO, C.M. \& C.C. Wang. 2000. Control of BTEX migration by intrinsic bioremediation at a gasoline spill site. Water Research 34(13): 3413-3423.

LaGreda, M.D., P.L. Buckinghan \& J.C. Evans. 1996. Gestión de residuos tóxicos. Tratamientos, eliminación, y recuperación de suelos. McGaw Hill/Interamerica de España, S.A. Vol. I: 1-642.

LEMLY, A.D. \& R.H. HILDERBRAND. 2000. Influence of large woody on stream insect community and benthic detritus. Hydrobiologia 421:179-185.

Mariuzzo, D.M. \& R.P. ScAmparinI. 1999. Polynuclear aromatic hydrocarbons biodegradation by indigenous bacteria isolated from Sao Paulo State rivers. IFT Annual meeting. http:// www.confex 2.com/ift/99annual/abstracts/ 4161.html.

Munné, A., C. Solá \& N. Prat. 1998. QBR: Un índice rápido para la evaluación de la calidad de los ecosistemas de ribera. Tecnología del Agua 175: 20-37.

Munné, A., N Prat, C. Solà, N. Bonada \& M RieradeVAll. 2003. A simple field method for assessing the ecological quality of riparian habitat in rivers and streams. QBR index. Aquatic 
Evaluación a través de indices QBR e IHF: PALMA, A. ET AL.

Conservation: Marine and Freshwater Ecosystems, 13: 147-164.

Naiman, R.J., H. Decamps \& M. Pollock.1993. The role of riparian corridors in maintaining biodiversity. Ecological Applications 2: 209-212.

Pardo, I., M. Álvarez, J. Casas, J. Moreno, S. Vicas, N. Bonada, J. Alba-Tercedor, P. Jáimez-Cuellar, G. Moya, N. Prat, S. Robles, M. Suarez, M. Toro \& M. Vidal-Abarca. 2002. El hábitat de los ríos mediterráneos. Diseño de un índice de diversidad de hábitat. Limnetica 21: 115-132

Rabeni, C.F. \& G.W. Minshall. 1977. Factors affecting microdistributions of stream benthic insects. Oikos 29: 33-43.

Ríos, S.L. \& R.C. BAILEy. 2006. Relationship between riparian vegetation and stream benthic communities at three spatial scales. Hydrobiologia 553: $153-160$.
Suarez, M.L, M.R. Vidal-Abarca, M. Sanchez-Montoya, J. Alba-Tercedor, M. Álvarez, J. Avilés, N. Bonada, J. Casas, P. Jáimez-Cuellar, A. Munné, I. Pardo, N. Prat, M. RieradeVall, M.J. Salinas, M. Toro \& S. Vivas. 2002. Las riberas de los ríos mediterráneos y su calidad: El uso del índice QBR. Limnetica 21: 135-148

Voelz, N.J. \& J.V. McArthur. 2000. An exploration of factors influencing lotic species richness. Biodiversity and Conservation 9: 1543-1570.

Wallace, J.B., S.L. EgGert, J.L. Meyer \& J.R. Webster. 1997. Multiple trophic levels of a forest stream linked to terrestrial litter inputs. Science 277: 102-104.

Ward, J.V., C.T. Robinson \& K. Tockner. 2002. Applicability of ecological theory to riverine ecosystems. Verthandlundgen der Internationale Vereinigung für theoretische und angewandte Limnologie 28: 442-450.

Recibido: 21.08.08

Aceptado: 18.05.09 